\title{
Observation of Algebraic Decay of Positional Order in a Smectic Liquid Crystal
}

Als-Nielsen, Jens Aage; Litster, J. D.; Birgeneau, R. J.; Kaplan, M.; Safinya, C. R.; Lindegaard-Andersen, Asger; Mathiesen, $\mathbf{S}$.

Published in:

Physical Review B

Link to article, DOI:

10.1103/PhysRevB.22.312

Publication date:

1980

Document Version

Publisher's PDF, also known as Version of record

Link back to DTU Orbit

Citation (APA):

Als-Nielsen, J. A., Litster, J. D., Birgeneau, R. J., Kaplan, M., Safinya, C. R., Lindegaard-Andersen, A., \& Mathiesen, S. (1980). Observation of Algebraic Decay of Positional Order in a Smectic Liquid Crystal. Physical Review B, 22(1), 312-320. https://doi.org/10.1103/PhysRevB.22.312

\section{General rights}

Copyright and moral rights for the publications made accessible in the public portal are retained by the authors and/or other copyright owners and it is a condition of accessing publications that users recognise and abide by the legal requirements associated with these rights.

- Users may download and print one copy of any publication from the public portal for the purpose of private study or research.

- You may not further distribute the material or use it for any profit-making activity or commercial gain

- You may freely distribute the URL identifying the publication in the public portal 


\title{
Observation of algebraic decay of positional order in a smectic liquid crystal
}

\author{
J. Als-Nielsen and J. D. Litster* \\ Riso National Laboratory. DK-4000 Roskilde, Denmark \\ R. J. Birgeneau, M. Kaplan, and C. R. Safinya \\ Department of Physics, Massachusetts Institute of Technology, \\ Cambridge. Massachusetts 02139 \\ A. Lindegaard-Andersen and S. Mathiesen \\ Technical Physics Laboratory III. Technical University of Denmark, \\ DK-2800 Lyngby, Denmark \\ (Received 17 September 1979)
}

\begin{abstract}
A smectic- $A$ liquid crystal in three dimensions has been predicted to exhibit algebraic decay of the layer correlations rather than true long-range order. As a consequence, the smectic Bragg peaks are expected to be power-law singularities of the form $q_{\|}^{-2+\eta}$ and $q_{\perp}^{-4+2 \eta}$, where $\|(\perp)$ is along (perpendicular to) the smectic density wave vector direction, rather than $\delta$ function peaks. Observation of these phenomena requires very high instrumental resolution together with a resolution function with wings which drop off much more rapidly than $q_{\|}^{-2}\left(q_{\perp}^{-4}\right)$. We show that these requirements may be met by using a three crystal $x$-ray spectrometer with multiplereflection channel cut crystals as monochromator and analyzer. We find that the smectic- $A$ Bragg peaks observed in the liquid-crystal octyloxy-cyanobiphenyl are indeed consistent with the predicted power-law singularity form. Furthermore, the explicit values of $\eta$ required to describe the measured profiles are in accordance with calculations of $\eta$ using the harmonic approximation with empirically determined splay and layer compressibility elastic constants.
\end{abstract}

\section{INTRODUCTION}

It has been known theoretically for some time that translational order as it occurs in a solid cannot exist in two dimensions because it is destroyed by thermally excited fluctuations. ${ }^{1,2}$ Thus for crystalline solids $d^{0}$, the lower marginal dimensionality, is two. This is the spatial dimension at which thermal fluctuations prevent the establishment of the long-range order which the interactions between the atoms would favor. For many systems $d^{0}$ is two, and this is one of the reasons for the intense recent experimental and theoretical interest in two-dimensional materials. For these systems it is predicted that a transition occurs to a state of quasi-long-range order in which the positional correlation functions do not extend to infinity, but decay algebraically as some power of the distance. $^{3}$ A similar algebraic decay of correlation functions occurs at an ordinary critical point and in both cases the power-law decay of correlations is accompanied by an infinite susceptibility. For various experimental reasons, this predicted behavior has not yet been observed directly in any two-dimensional system. ${ }^{4}$

A closely related phenomenon is predicted to occur in smectic- $A$ and $-C$ liquid crystals. ${ }^{5,6}$ These are phases of liquid crystals which possess both orientational long-range order (LRO) of the anisotropic molecules and translational-order intermediate between that of a liquid and a solid. ${ }^{6}$ Explicitly, in the $A$ and $C$ phases one has a one-dimensional mass-density wave in a three-dimensional liquid. The density wave may be either along ( $A$ phase) or at an angle $\phi$ ( $C$ phase $)$ to the nematic director. As we shall discuss below, it may be readily shown that the positional fluctuations diverge logarithmically at large distances at all temperatures in exact analogy with $2 \mathrm{D}$ crystals. Thus the smectic- $A$-to-nematic phase transition is not only a relatively simple example of melting, but such liquid crystals also provide a threedimensional system in which the effects of divergent long-wavelength acoustic fluctuations can be studied experimentally. Ultimately one may hope that study of smectic phases will lead to better understanding of melting, a problem which still eludes condensedmatter physicists.

In this paper we report the results of a high-resolution $x$-ray-diffraction study of the density wave in the smectic- $A$ phase of octyloxy-cyanobiphenyl (8OCB).

The traditional picture ${ }^{6}$ of the smectic- $A$ phase $(\mathrm{SmA})$ is shown in the left part of Fig. 1. The molecular centers are arranged in layers with a well- 


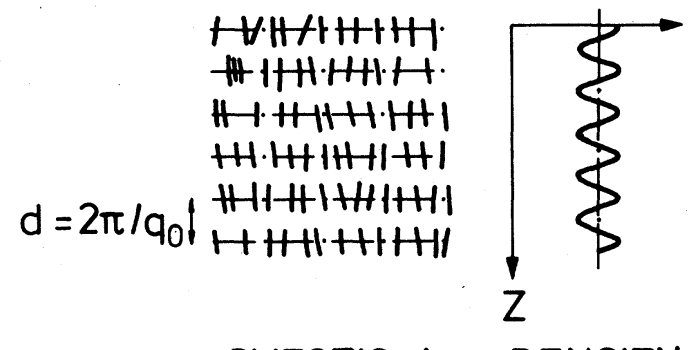

SMECTIC A: DENSITY

FIG. 1. The smectic- $A$ phase consists of a one-dimensional density wave along the average direction of the molecular axis. The sinusodial shape of the density wave is reflected in the diffraction pattern by the absence of higherorder reflections.

defined layer spacing $d$ but with a liquidlike order within each layer. The average orientation, $\vec{n}$, of the molecules is perpendicular to the layers in the SmA phase. If this picture were literally correct, Bragg scattering of $x$-rays should occur whenever the difference between incident and scattered wave vectors equals a multiple of $q_{0}=2 \pi / d$, that is $\overrightarrow{\mathrm{k}}_{i}-\overrightarrow{\mathrm{k}}_{f}=p(2 \pi / d) \overrightarrow{\mathrm{n}}$ with $p=1,2 \ldots$. In an earlier study ${ }^{7}$ we found that very near $T_{c}$ the higherorder Bragg peaks are absent, or precisely, their intensity is at least a factor of $10^{4}$ less than the fundamental reflection. A more correct description of the $\mathrm{SmA}$ phase is therefore that the density forms a sinusoidal ripple as shown in the right-hand part of Fig. 1, and the dot-dashed lines through the molecules should not be interpreted as lattice planes but rather as planes of a certain phase of the density wave. The question is now whether this ripple has true long-range order.

Let us briefly recall the arguments of Landau ${ }^{2}$ and Lifshitz $z^{5}$ and by Peierls ${ }^{1}$ on this point. The longwavelength acoustic modes shown pictorially in Fig. 2 arising from the SmA ordering involve a displacement $u$ of the smectic layers in the $z$ direction, or more properly gradients in the phase $\phi=q_{0} u$ of the density wave. These modes have unusually anisotropic elastic behavior. With the wave vector $\overrightarrow{\mathrm{q}}$ along $z$
( $\overrightarrow{\mathrm{q}}=q_{\|} \hat{z}$, see upper part of Fig. 2) the displacement $u$ is longitudinal, and the energy is of the usual elastic form $\frac{1}{2} B_{q_{\|}} u^{2}(\overrightarrow{\mathrm{q}})$ with $B$ a compressibility for the smectic layers. However with $\vec{q}$ normal to $\hat{z}$, the displacement is transverse and the layer separation is unchanged as is evident from the lower part of Fig. 2 ; thus, to second order in $q_{1}$, the displacement requires no elastic energy. The restoring force for $q_{\|}=0$ then arises because the director field $\vec{n}$ remains normal to the layers and thus a director splay distortion results with an elastic energy density $\frac{1}{2} K(\vec{\nabla} \cdot \overrightarrow{\mathrm{n}})^{2}$, where $K$ is the nematic phase splay elastic constant. Since $\overrightarrow{\mathrm{n}}$ is normal to the layers, one has $\delta \overrightarrow{\mathrm{n}}=-\vec{\nabla}_{\perp} u$ and an elastic energy $\frac{1}{2} K q_{\perp}^{4} u^{2}(\overrightarrow{\mathrm{q}})$. Experimentally one finds that $K$ has the same value in both the nematic and smectic phases near the SmA-N transition. ${ }^{8}$ Since a magnetic field exerts a torque on the molecules, the complete expression for the elastic energy density of the SmA elastic modes of wave vector $\vec{q}$ is

$$
F_{\vec{q}}=\frac{1}{2}\left(B q_{\|}^{2}+\chi_{a} H^{2} q_{\perp}^{2}+K q_{\perp}^{4}\right) u^{2}(q),
$$

where $\chi_{a}$ is the volume diamagnetic susceptibility anisotropy that results from nematic ordering. We note that $B$ and $\chi_{a} H^{2}$ have the dimensions of energy per unit length. The characteristic lengths of the smectic phase are thus the sample dimension $L$, the density wavelength $2 \pi / q_{0}$, and two additional lengths $\lambda=(K / B)^{1 / 2}$ and $\xi_{M}=\left(K / \chi_{a} H^{2}\right)^{1 / 2}$. Both $\lambda$ (the penetration depth) and $\xi_{M}$ (the magnetic coherence length) are relaxation lengths; $\lambda$ determines the decay of an undulation distortion (pure splay director distortion) imposed at the surface of a uniform smectic. A surface-imposed director alignment in a nematic phase will relax to that favored by a magnetic field with a characteristic distance $\xi_{M}$; in the smectic- $A$ phase $\xi_{M}$ determines the long-wavelength cutoff of the diverging fluctuations in $u$.

The mean-squared fluctuations $\left\langle u^{2}\right\rangle$ can be calculated by applying the equipartition theorem to Eq. (1) and summing over all wave vectors $<q_{0}$. One readily finds

$$
\begin{aligned}
\left\langle u^{2}\right\rangle & =(2 \pi)^{-3} k T \int_{-q_{0}}^{q_{0}} d q_{\|} \int_{q_{\min }}^{q_{\max }}\left(B q_{\|}^{2}+\chi_{a} H^{2} q_{\perp}^{2}+K q_{\perp}^{4}\right)^{-1} 2 \pi q_{\perp} d q_{\perp} \\
& =(4 \pi)^{-1} k T(B K)^{-1 / 2} \int_{q_{\min }}^{q_{\max }}\left(q_{\perp}^{2}+\xi_{M}^{-2}\right)^{-1 / 2} d q_{\perp} \\
& =(4 \pi)^{-1}(B K)^{-1 / 2} k T \ln \left(q_{\max } L / 2 \pi\right) \text { for } \xi_{M}=0 \\
& \simeq(4 \pi)^{-1}(B K)^{-1 / 2} k T \ln \left(2 \xi_{M} q_{\max }\right) \text { for } 0<\xi_{M}<L L .
\end{aligned}
$$

The mean-squared displacement thus diverges logarithmically with the smaller of $L$ or $\xi_{M}$ and, in this model, an infinite three-dimensional smectic- $A$ liquid crystal in zero field does not have true long-range positional order at any finite temperature. A similar divergence due to long-wavelength modes makes two-dimensional crystals 


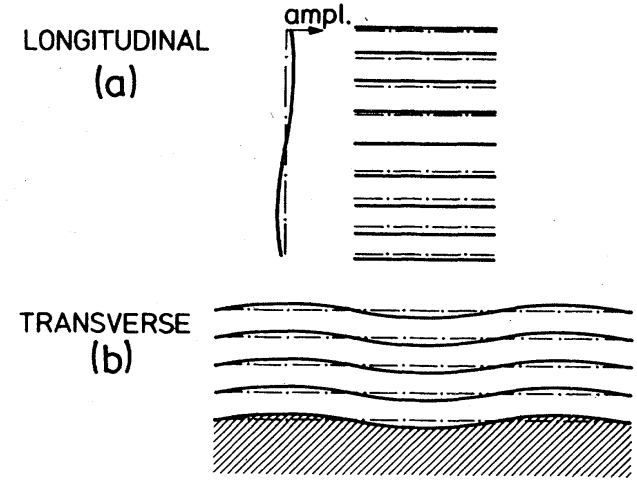

FIG. 2. (a), (b) The two fundamental long-wavelength modes of the smetic- $A$ phase. The energy density is proportional to $q_{\|}^{2}$ for the longitudinal mode but to $q_{1}^{4}$ for the transverse mode.

unstable. We should note, however, that there are a number of important differences in the predicted phase transition behavior of these two systems. For example, the 2D crystal should exhibit no observable specific-heat singularity on melting whereas the 3D smectic- $A$ exhibits a dramatic $\lambda$ anomaly in its specific heat at the N-A transition. ${ }^{9}$ There is not yet a fully satisfactory theory for the nematic-smetic- $A$ transition. However, in the approach presented by Halperin and Lubensky ${ }^{10}$ one may define a nonlocal order parameter which has true LRO in three dimensions in spite of the fact that the positional correlations are expected to decay algebraically with distance in the smectic- $A$ phase.

The consequences of the divergent real-space positional fluctuations for $x$-ray scattering may be calculated in the harmonic approximation. ${ }^{11}$ The details of this calculation are given in Appendix A. In this case the instantaneous correlations exhibit an anisotropic power-law dependence on molecular separation. On Fourier transforming for a sample in zero field, one finds that the $\mathrm{x}$-ray scattering is predicted to follow

$$
S\left(0,0, q_{\|}\right) \sim\left(q_{\|}-q_{0}\right)^{-2+\eta}
$$

and

$$
S\left(q_{\perp}, 0, q_{0}\right) \sim q_{\perp}^{-4+2 \eta}
$$

with

$$
\eta=k T\left(q_{0}^{2} / 8 \pi\right)(B K)^{-1 / 2} .
$$

We should emphasize that in $x$-ray scattering we measure the instantaneous correlations so that Eq. (3) represents quasielastic rather than true elastic scattering. Analogous results have been obtained for the two-dimensional harmonic crystal. ${ }^{12-14}$ Near the nematic-smectic transition temperature $T_{c}, B$ becomes very small ${ }^{8}$ and the harmonic approximation may be expected to fail; however there is as yet no theory for this region. As can be seen from Eq. (2), finite sample dimensions or a magnetic field remove the logarithmic singularity. In principle these will cause true Bragg scattering as well as the quasielastic scattering of Eq. (3); however this finite size scattering will be indistinguishable from Landau-Peierls scattering if the wave vector of the longest wave fluctuations is much less than the instrumental resolution.

\section{EXPERIMENTAL}

Experimentally it is not trivial to distinguish between the "Landau-Peierls" scattering of Eq. (3) and a combination of Bragg scattering plus thermal diffuse scattering from the acoustic modes. The instrumental resolution must be very good to distinguish between a $\delta$ function (Bragg) and a cusp (Landau-Peierls). High angular resolution can be obtained by using perfect crystals together with an essentially monochromatic $x$-ray line source; only $x$ rays collimated within a few milliradians will fulfill the Bragg condition. In an earlier study we used the (111) reflection from perfect $\mathrm{Ge}$ crystals to define the direction of the incident and scattered $x$ rays. In this study we did not fully appreciate the extent to which tails of the resolution function, arising primarily from extinction, ${ }^{15}$ made it impossible to conclude unambiguously that the tails we observed were indeed caused by the Landau-Peierls scattering.

The solution to this problem is to use multiple Bragg reflections in the collimating crystals, since the resolution after $m$ Bragg reflections is that for a single reflection raised to the $m$ th power. ${ }^{16}$ In the present paper we have investigated the resolution functions in great detail and are able to prove that the $\mathrm{SmA}$ line profile is consistent with Landau-Peierls scattering as described by Eq. (3) with essentially no adjustable parameters.

The high-resolution experimental setup at $R$ is $\varnothing$ is shown schematically in Fig. 3. The scattering plane is

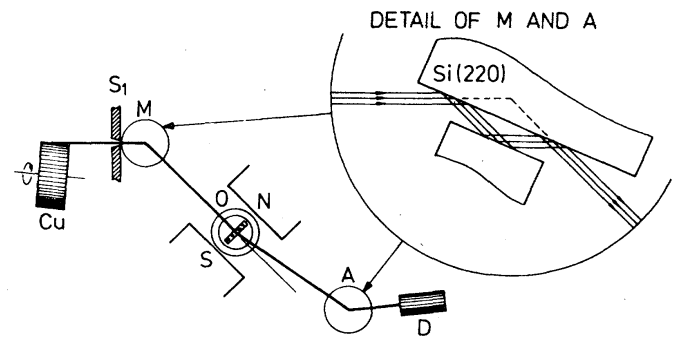

FIG. 3. Experimental setup using triple-reflection channel-cut crystals as monochromator $(M)$ and analyzer $(A)$ crystals. A single-domain smectic- $A$ sample is obtained by aligning the director by a magnetic field. $S_{1}$ is the slit to separate $K \alpha_{1}$ from $K \alpha_{2}$ lines; $O$ is a two-stage oven holding the sample; $D$ is a scintillation detector. 
horizontal and the $\mathrm{x}$-ray source has dimensions 0.9 $\mathrm{mm}$ wide by $0.6 \mathrm{~mm}$ high seen from the monochromator $M$. The $\mathrm{x}$-ray generator is a rotating-anode machine operating at $55 \mathrm{kV}$ and $180 \mathrm{~mA}$ with a copper target. A $0.5-\mathrm{mm}$ wide $3-\mathrm{mm}$ high slit $S_{1}, 65$ $\mathrm{cm}$ from the source enables the monochromator to separate the $K \alpha_{1}$ and $K \alpha_{2}$ lines. Because the incident beam is depleted by scattering as it penetrates the monochromator crystal, only a finite number of crystal planes contribute to the Bragg scattering. Thus even a perfect crystal with a monochromatic $x$ ray beam will have a finite angular acceptance known as the Darwin width $w_{D}$; and the beam incident on the sample has an angular divergence equal to the Darwin width. The theoretical value of $w_{D}$ for the $\mathrm{Si}$ (220) reflections we used is $0.0012^{\circ}$. The direction of $\mathrm{x}$ rays scattered by the sample is similarly determined by the analyzer $A$. A two-stage oven located at $0,44 \mathrm{~cm}$ from the monochromator, contains the 1.5-mm-thick flat $80 C B$ sample between Be windows; to obtain a single domain sample a field of $4.8 \mathrm{kG}$ was supplied by an electromagnet. The analyzer was 36 $\mathrm{cm}$ from the sample, and a slit $(4 \mathrm{~mm}$ wide, $13 \mathrm{~mm}$ high) determined the illuminated area of the scintillation detector $D$. The channel-cut crystals used for $M$ and $A$ are discussed in more detail in an Appendix.

\section{LINE PROFILE OF BRAGG SCATTERING}

In this section we shall consider in some detail the Bragg line shape one would have observed if the SmA phase had true long-range order. As we shall see one can, by simple scaling of a measured Bragg profile which in our case originated from a $\mathrm{Si}(111)$ reflection, simulate the Bragg profile of a crystal with the same planar spacing as $80 \mathrm{CB}$. Furthermore, we account in detail for the $\mathrm{Si}(111)$ profile from measured properties of the monochromator and analyzer crystals. In the end of the section we compare the simulated Bragg profile with the actual line profile from the $\mathrm{SmA}$, and this comparison shows that the SmA phase exhibits anomalously intense diffuse scattering which is consistent with our expectations based on the harmonic theory but which appears to be much too strong to be accounted for on the basis of normal thermal diffuse scattering accompanying a true Bragg peak.

There are two contributions to the width, or in general the shape, of a Bragg profile. One is the Darwin width of the perfect monochromator and analyzer crystals as already discussed in the previous section; the other is the spectral width of the $\mathrm{Cu} K \alpha_{1}$ line. In order to discuss the latter effect let us, as illustrated in Fig. 4, consider two wavelengths, $\lambda$ and $\lambda+\Delta \lambda$, as they are Bragg scattered by the monochromator $M$, the sample $S$, and the analyzer $A$. The difference in Bragg angles between the two wave-

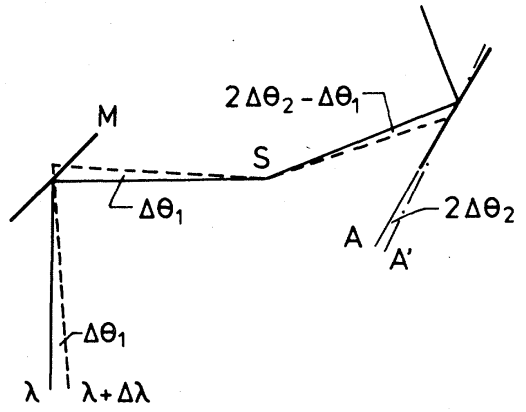

NONDISPERSIVE

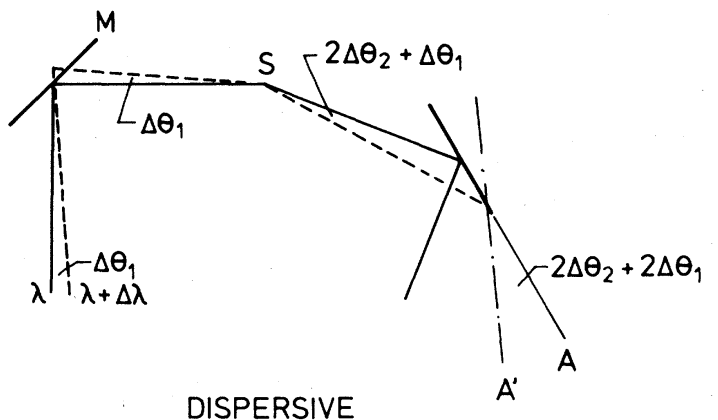

FIG. 4. The geometry of the dispersive (bottom) and nondispersive (top) orientation of the monochromator $(M)$ and analyzer $(A)$ crystals and sample $(S)$. Note that the dashed lines representing rays with wavelength $\lambda+\Delta \lambda$ will only be reflected by the anlayzer crystal if either $2 \Delta \theta_{2}$ or $2 \Delta \theta_{2}+2 \Delta \theta_{1}$ is less than the Darwin width for the nondispersive and dispersive configurations, respectively. Therefore as the sample scattering angle $2 \theta_{2}$ becomes small relative to the analyzer Bragg angle $2 \theta_{1}$, the resolution function in the nondispersive configuration approaches the direct beam profile.

lengths is $\Delta \theta=(\Delta \lambda / \lambda) \tan \theta$. We use subscript 1 for Bragg angles in the identical monochromator and analyzer crystals, and subscript 2 for the Bragg angle in the sample. It is important to notice the difference between the two possible analyzer orientations as illustrated in the top part and in the bottom part of Fig. 4. In the first case the analyzer must be turned through the angle $2 \Delta \theta_{2}$ counter clockwise in order to scatter the wavelength $\lambda+\Delta \lambda$, whereas it must be turned the angle $2 \Delta \theta_{2}+2 \Delta \theta_{1}$ clockwise in the latter case. Suppose there is no sample. In the top part of Fig. 4 the analyzer should then not be turned at all in order to scatter $\lambda+\Delta \lambda$, and scanning the analyzer would not yield any information about the wavelength distribution. But in the setting of the bottom part of Fig. 4 an analyzer scan determines directly the intrinsic linewidth of the $\mathrm{Cu} K \alpha_{1}$ line, the angular width being $2(\Delta \lambda / \lambda) \tan \theta$, provided that the width is much larger than $w_{D}$. This orientation of monochromator and analyzer is called the dispersive orienta- 


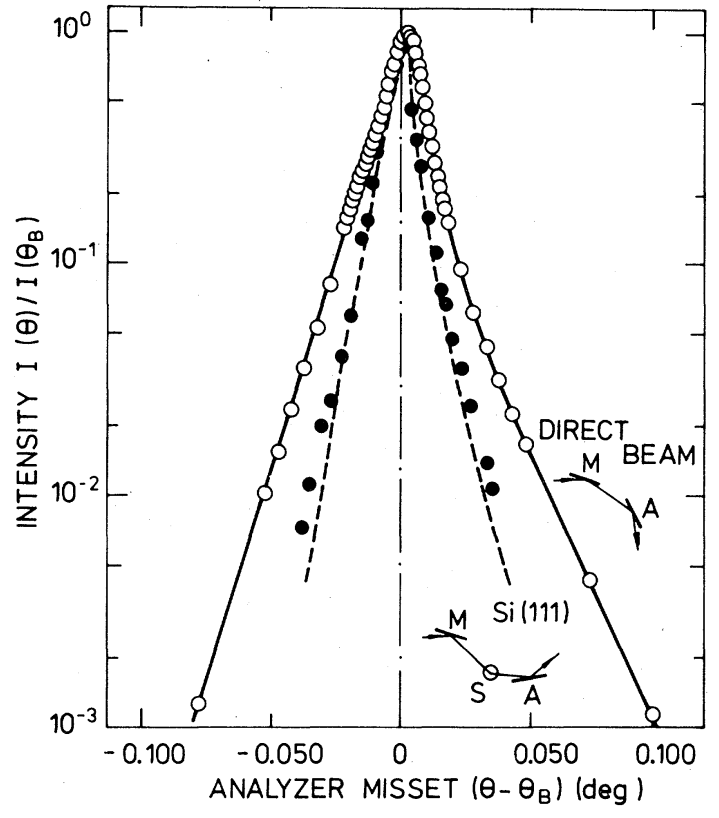

FIG. 5. Direct beam profile in the dispersive orientation (open circles) and the profile of a Si(111) reflection in the nondispersive orientation (filled circles). The solid line is a guide to the eye. The dashed line is the resolution function as calculated from the direct beam profiles in the dispersive and nondispersive orientation.

tion. We have examined the resolution function of the apparatus by analyzer scans with no sample for both the dispersive and nondispersive orientations. With scattering through a finite angle by a sample, the $\mathrm{x}$-ray linewidth broadens the resolution even for the nondispersive orientation. From the direct beam profile with the analyzer in the dispersive orientation (shown as open circles in Fig. 5) we find a full width at half maximum (FWHM) $0.015^{\circ}$ or $0.262 \mathrm{mrad}$. With the Bragg angle $\theta_{1}=23.651^{\circ}$ we find $\Delta \lambda / \lambda=3.1$ $\times 10^{-4}$ for the $\mathrm{Cu} K \alpha_{1}$ line. We can then use this result to calculate the line shape for Bragg scattering from a sample with nondispersive orientation. For $\mathrm{Si}(111)$, neglecting the finite Darwin width, we should expect to scale the abscissa by $\tan \theta_{111} / \tan \theta_{220}$ $=0.579$; we find $w=0.00897^{\circ}$ FWHM. Since $w^{2} \gg w_{D}^{2}$, neglect of the Darwin width was a good approximation. The predicted $\mathrm{Si}(111)$ line (dashed curve) and the data points (solid circles) agree quite well. It should be noted that if the sample is a single crystal with a narrow mosaic spread, the sample must be rotated along with the analyzer to scan the Bragg peak. In the nondispersive orientation the ratio of sample to analyzer rotations is $-2 \tan \theta_{2} /\left(\tan \theta_{1}-\tan \theta_{2}\right)$ $=-2.75$, the minus sign indicating rotations in opposite directions.

The direct beam profile for nondispersive orientations is shown (open circles) in Fig. 6. The FWHM is not far from the theoretical limit $w_{D}=0.0012^{\circ}$.

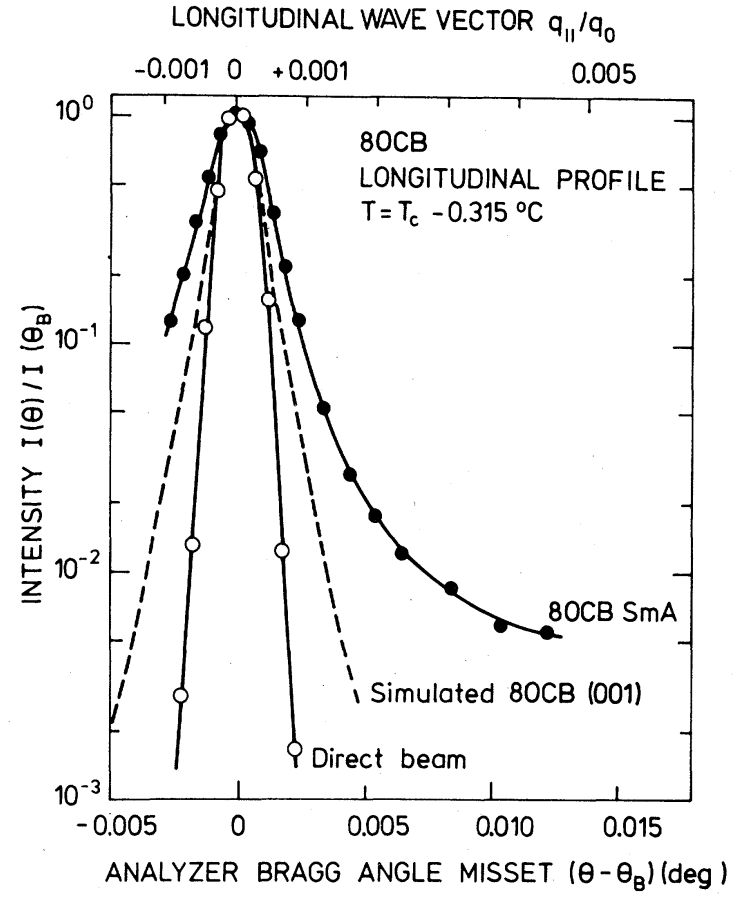

FIG. 6. The calculated and measured line profile of $\mathrm{Si}(111)$ gives by simple scaling a simulation of the $8 \mathrm{OCB}$ profile if the smectic- $A$ phase had true long-range order (dashed line). This profile is somewhat broader than the direct beam profile (open circles) due to the linewidth of the $\mathrm{Cu} K \alpha_{1}$ line. The measured profile from 8OCB (filled circles) exhibits pronounced wings, and the full line is a leastsquares fit of the theoretical line shape discussed in the text folded with the experimental resolution.

More important is the very rapid falloff in intensity as the angular misset exceeds $w_{D}$. This tailless resolution is the virtue of channel-cut crystals as discussed in the Appendix. We may calculate the expected line profile for a true Bragg peak corresponding to $q_{0}$ for $8 \mathrm{OCB}$ (which has $\theta_{2}=1.39^{\circ}$ ). The result is obtained by scaling the abscissa for the $\mathrm{Si}(111)$ peak of Fig. 5 by $\tan \left(1.39^{\circ}\right) / \tan \left(14.221^{\circ}\right)$ $=0.096$, to obtain the width due to $\Delta \lambda$ and then convoluting this with the profile determined by scanning through the forward direction in the nondispersive orientation; the net result is shown as a dashed line in Fig. 6. The line profile observed at a reduced temperature $t=1-T / T_{c}=9 \times 10^{-4}$ is shown as solid circles in Fig. 6. We see this differs significantly from what one would expect for a conventional Bragg peak, the difference being more than an order of magnitude at a misset of $0.005^{\circ}$ from the peak. We emphasize that the expected profile for true Bragg scattering is obtained by direct scaling in angle of experimental data [for the $\mathrm{Si}$ (111) Bragg peak] that is, with this ultrahigh resolution, normal thermal diffuse scattering in the silicon is almost undetectable. 


\section{QUANTITATIVE DATA ANALYSIS}

In our experiment we measure the convolution of the scattering $S(\overrightarrow{\mathrm{q}})$ with the spectrometer resolution function $R(\overrightarrow{\mathrm{q}})$. Thus if the spectrometer is set for wave-vector transfer $\vec{q}$, a variety of scattering processes in the vicinity of $\vec{q}$ will be picked up as

$$
S(\overrightarrow{\mathrm{q}}) \sim \int S\left(\overrightarrow{\mathrm{q}}^{\prime}\right) R\left(\overrightarrow{\mathrm{q}}-\overrightarrow{\mathrm{q}}^{\prime}\right) d \overrightarrow{\mathrm{q}}^{\prime} .
$$

A conventional deconvolution by direct numerical integration is quite inefficient because $S(\overrightarrow{\mathrm{q}})$ diverges at $\left(0,0, q_{0}\right)$. Fortunately we have an explicit theoretical expression for $S(\overrightarrow{\mathrm{r}})$, the Fourier transform of $S(\overrightarrow{\mathrm{q}})$, rather than for $S(\overrightarrow{\mathrm{q}})$ itself. We may therefore use the folding theorem to calculate

$$
S(\overrightarrow{\mathrm{q}})=(2 \pi)^{3} \int d \overrightarrow{\mathrm{r}} S(\overrightarrow{\mathrm{r}}) F(\overrightarrow{\mathrm{r}}) e^{i \overrightarrow{\mathrm{q}} \cdot \overrightarrow{\mathrm{r}}},
$$

where $F(\overrightarrow{\mathrm{r}})$ is the Fourier transform of $R(\overrightarrow{\mathrm{q}})$ and the correlation function $S(\vec{r})$ was calculated by Caillé $^{11}$ (see Appendix) as

$S(\overrightarrow{\mathrm{r}}) \sim e^{-2 \gamma \eta}\left(\frac{1}{x^{2}+y^{2}}\right)^{\eta} \exp -\left[\eta E_{1}\left(\frac{x^{2}+y^{2}}{4 \lambda z}\right)\right]$

with $\lambda=(K / B)^{1 / 2}$ and $\eta$ given by Eq. (3c). The resolution in the vertical $(y)$ direction can be calculated from the geometry shown in Fig. 3. It is actually trapezoidal with base full width 20 mrad and peak full width 11 mrad; we approximate it for ease of calculation with a Gaussian of the same FWHM $\left(\sigma_{y}=0.22 q_{0}\right.$, where $\left.q_{0}=0.197 \AA^{-1}\right)$. The longitudinal resolution (along $q_{z}=q_{11}$ ), is given by the dashed line in Fig. 6; it has tails which are very nearly exponential. We therefore represented it within the accuracy of our measurements by the best fit to the convolution of a Gaussian with an exponential; the functional form is given by Table I and the parameters are $\sigma_{z}=2.2 \times 10^{-4} q_{0}$ with the momentum-space decay of the exponential tail being $\sigma \sigma_{z}=1.54 \sigma_{z}$. This resolution function has a FWHM of $0.0014^{\circ}$, only slightly larger than the theoretical Darwin width. The exponential wings of the longitudinal resolution

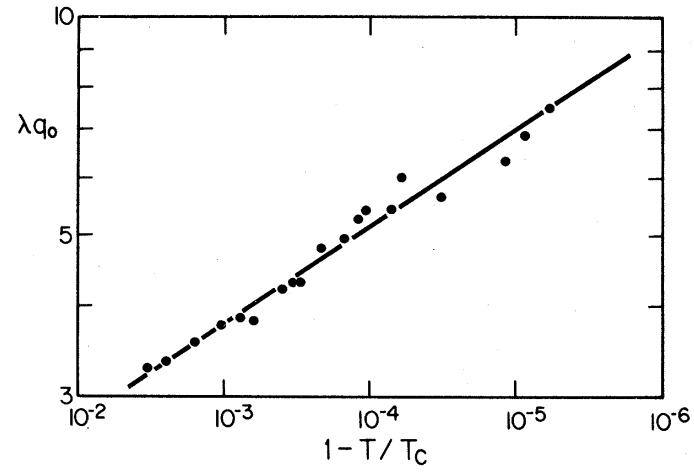

FIG. 7. Liquid-crystal penetration depth, $\lambda=(K / B)^{1 / 2}$, in the smectic- $A$ phase of $80 C B$. As $T$ approaches $T_{c}$ the layer stiffness constant $B$ approaches zero. The solid line is the equation $\lambda q_{0}=1.55\left(1-T / T_{c}\right)^{-0.13}$ obtained from a leastsquares fit to a power law. The data are from Ref. 17.

function are an experimental fact which we do not fully understand. Finally, the transverse in-plane resolution was determined by the mosaicity of the liquid-crystal sample, which showed some variation with temperature. We represented the mosaic distribution by a Gaussian whose width was determined by a fit to points above $20 \%$ peak intensity for sample rocking curves $\left(q_{\perp}=q_{x}\right.$ scans) for each temperature. The results were: $\sigma_{x} / q_{0}=6.45 \times 10^{-3}, 4.2 \times 10^{-3}$, and $4.9 \times 10^{-3}$ for $t=9.0 \times 10^{-4}, 5.9 \times 10^{-4}$, and $4 \times 10^{-6}$, respectively. The reciprocal-space resolution function is given by $R(\overrightarrow{\mathrm{q}})=R_{1}\left(q_{x}\right) R_{2}\left(q_{y}\right) R_{3}\left(q_{z}\right)$, and its real-space transform is $F(\vec{r})=F_{1}(x) F_{2}(y) F_{3}(z)$; all of these functions are given in Table $I$. The data analysis consisted of a nonlinear least-squares fit of the Fourier transform of $F(\vec{r})$ to the longitudinal scan data normalized to the peak height. Values of $q_{\|}<0.999 q_{0}$ were not used because of contaminant scattering from the $K \alpha_{2}$ line. $S(\overrightarrow{\mathrm{r}})$ is determined by two parameters: $\lambda$ and $\eta$. Since the penetration depth has been accurately measured by light scattering, ${ }^{8,17}$ we used these results in our data analysis. The measured value of $\lambda q_{0}$ for 8OCB is shown in Fig. 7. The fits were therefore carried out with only

TABLE I. The experimental resolution function (values for the parameters are given in the text).

\begin{tabular}{ll}
\hline \hline \multicolumn{1}{c}{ Momentum space } & Real space \\
\hline$R(\overrightarrow{\mathrm{q}})=R_{1}\left(q_{x}\right) R_{2}\left(q_{y}\right) R_{3}\left(q_{z}\right)$ & $F(\vec{r})=F_{1}(x) F_{2}(y) F_{3}(z)$ \\
$R_{1}\left(q_{x}\right)=\exp \left(-q_{x}^{2} / 2 \sigma_{x}^{2}\right)$ & $F_{1}(x)=\exp \left(-x^{2} \sigma_{x}^{2} / 2\right)$ \\
$R_{2}\left(q_{y}\right)=\exp \left(-q_{y}^{2} / 2 \sigma_{y}^{2}\right)$ & $F_{2}(y)=\exp \left(-y^{2} \sigma_{y}^{2} / 2\right)$ \\
$R_{3}\left(q_{z}\right)=\int \exp \left(-q_{1}^{2} / 2 \sigma_{z}^{2}\right)$ & $F_{3}(z)=\frac{\exp \left(-z^{2} \sigma_{z}^{2} / 2\right)}{\left(1+\sigma^{2} \sigma_{z}^{2} z^{2} / 2\right)}$ \\
$\quad \times \exp \left(\sqrt{2}\left|q_{z}-q_{1}\right| / \sigma \sigma_{z}\right) d q_{1}$ & \\
\hline
\end{tabular}


TABLE II. Exponent $\eta$ and elastic constant $K$.

\begin{tabular}{clll}
\hline & & & \\
$t$ & $9.0 \times 10^{-4}$ & $5.9 \times 10^{-4}$ & $4 \times 10^{-6}$ \\
$\eta$ & $0.17 \pm 0.02$ & $0.23 \pm 0.02$ & $0.38 \pm 0.06$ \\
$10^{7} K$ (dyne) & $8.4 \pm 1$ & $7.1 \pm 0.6$ & $7.7 \pm 2$ \\
& & & \\
\hline
\end{tabular}

one adjustable parameter, the exponent $\eta$. The results of the fits are shown in Table II. The uncertainties are those which result in a doubling of $\chi^{2}$ and include the effects of experimental errors in $\lambda$ and the resolution function. The resulting fits for two longitudinal scans are shown along with the longitudinal resolution function in Fig. 8 .

As a final check in our analysis, we give the elastic constant $K$ deduced from each value of $\eta$ in Table II. It has been established experimentally that $K$ shows no anomalous behavior near the $\mathrm{A}-\mathrm{N}$ transition; thus

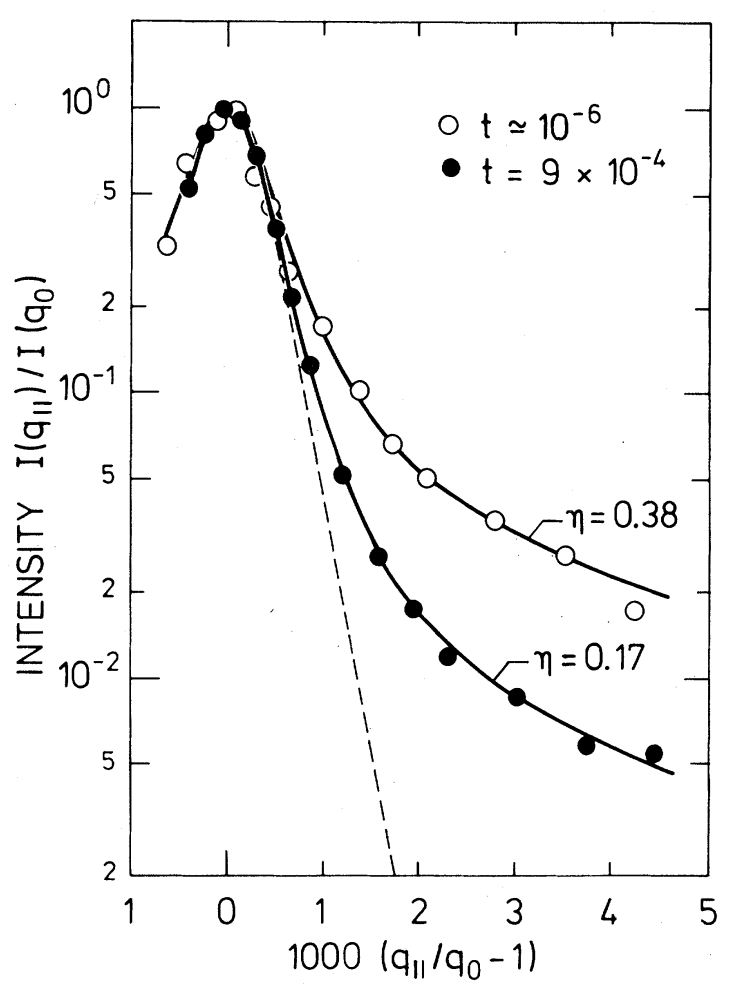

FIG. 8. Longitudinal line profiles at two reduced temperatures. The dashed line is the experimental resolution as shown and discussed in Fig. 6, the full line the best fit of the theoretical line shape $\left(q_{\|}-q_{0}\right)^{-2+\eta}$ folded with the threedimensional resolution function using the exponent $\eta$ and an overall scale factor as adjustable parameters. As discussed in the text the values of $\eta$ so-obtained agree with those calculated from the empirical values of $q_{0}, B$, and $K$.
$K$ should be constant over the narrow temperature range of our experiments. This is true within the experimental uncertainties. There are also independent measurements of $K$ reported in the literature. ${ }^{18}$ In these measurements a magnetic field induces a distortion in a liquid-crystal film (a Freedericks transition) of thickness $l$ at a threshold field when $\xi_{M}=1 / \pi$; thus a determination of $K$ requires a knowledge of $\chi_{a}$. Karat and Madhusudana ${ }^{18}$ report $K=1.8 \times 10^{-6}$ dyne, however they used an anisotropy in the 8OCB molar susceptibility $\Delta \chi_{M}=118.6 \times 10^{-6}$ $\mathrm{cm}^{3} /$ mole. This figure was obtained by an incorrect average of the principal values for the biphenyl molecule. Using known anisotropies ${ }^{19,20}$ for various elements of the 8OCB molecule we calculate $\Delta \chi_{M}=44 \times 10^{-6} \mathrm{~cm}^{3} /$ mole. Including errors in $\Delta \chi_{M}$ and the nematic order parameter at the N-A transition, we find the correct value for $K$ is $(6.8 \pm 0.7)$ $\times 10^{-7}$ dyne. This is in excellent argeement with the values we obtained in Table II. Substituting our measured values into Eq. (2a) we obtain $\left\langle u^{2}\right\rangle^{1 / 2}=17$ $\AA$ at $t=4 \times 10^{-6}$ for $L=1 \mathrm{~cm}$. In a field of $4.8 \mathrm{kG}$ at the same temperature we calculate $\xi_{M}=6 \times 10^{-4} \mathrm{~cm}$ and $\left\langle u^{2}\right\rangle^{1 / 2}=14 \AA$; this illustrates rather dramatically how slow is the logarithmic divergence of Eq. (2).

It should then be possible to use the parameters obtained from analysis of the longitudinal scans to calculate the transverse $\left(q_{x}\right)$ scans. We find excellent agreement with no adjustable parameters down to $10 \%$ of peak height, but at $1 \%$ of peak height the experimental data are $\sim 60 \%$ higher than we calculate. This is probably because the wings of the mosaic distribution fall off less rapidly than a Gaussian due to defects near the edges of the smectic sample. These defects (e.g., edge dislocations) are undoubtedly a small fraction of the total volume of the sample but can contribute to a broader mosaicity at the $1 \%$ level. A Gaussian mosaic spread is an excellent approximation to the transverse resolution used in deconvoluting longitudinal scans, but mosaicity must be very accurately known well below $1 \%$ of peak in order to analyze the transverse scans reliably.

In summary, then, the complete longitudinal profile can be accurately described using Caille's harmonic theory with essentially no adjustable parameters. Further, the theory gives a good account of the transverse scans, although there is a slight discrepancy at the $1 \%$ level, presumably due to our incomplete knowledge of the sample mosaic distribution. As expected, the exponent $\eta$ which characterizes the algebraic decay of the positional correlations increases markedly as one approaches $T_{c}$. Although we have not proven that the Caille theory is unique, we have certainly proven that it is completely consistent with all of the data and we believe that this constitutes convincing evidence that the SmA phase does indeed exhibit the expected Landau-Peierls lack of true positional LRO. 


\section{CONCLUSIONS}

The data of Fig. 6 show that the smectic- $A$ state is not an infinite stacking of planes with a well-defined lattice spacing. This is consistent with the theoretical model of the SmA state as described by the freeenergy density of Eq. (1). Furthermore, the x-ray line profiles can be evaluated in this model in the harmonic approximation in terms of independently determined parameters such as the penetration depth and the splay elastic constant. With no adjustable parameters, apart from an overall scale factor, we find excellent agreement between the theoretical line shape folded with the instrumental resolution and the experimental data within a temperature range where the exponent $\eta$ describing the algebraic decay of correlations varies by a factor of two. This evidence for having observed the quasi-long-range order associated with divergent long-wavelength acoustic fluctuations could be further substantiated by examining the profile of higher-order reflections as well as transverse profiles and further work along these lines is in progress.

\section{ACKNOWLEDGMENTS}

We would like to acknowledge helpful discussions with Y. Imry, D. E. Moncton, and M. J. Stephen. The $\mathrm{X}$-ray source and the spectrometer were granted by the Danish National Science Foundation. Work at M.I.T. was supported by the U. S. National Science Foundation Grant No. DMR-76-18035 and the Joint Services Electronics Program, Contract No. DAAG29-78-C-0020.

\section{APPENDIX A: THE CORRELALTION FUNCTION AND SCATTERING CROSS SECTION}

The $x$-ray-scattering cross section is proportional to the Fourier transform of the correlation function

$$
S(\overrightarrow{\mathrm{r}})=\left\langle\exp \left\{i q_{0}[u(r)-u(0)]\right\}\right\rangle .
$$

In the harmonic approximation $u(r)$ is a Gaussian random variable so $S(\overrightarrow{\mathrm{r}})$ is given by $\exp \left[-\frac{1}{2} q_{0}^{2}\right.$ $\left.\times\left\langle|u(r)-u(0)|^{2}\right\rangle\right]$. Thus we need to evaluate $\left\langle\frac{1}{2} q_{0}^{2}|u(\overrightarrow{\mathrm{r}})-u(0)|^{2}\right\rangle$

$$
=\frac{k T q_{0}^{2}}{(2 \pi)^{3} B} \int \frac{[1-\cos (\overrightarrow{\mathrm{q}} \cdot \overrightarrow{\mathrm{r}})] d \overrightarrow{\mathrm{q}}}{\left(q_{\|}^{2}+\lambda^{2} q_{\perp}^{4}\right)}
$$

with $\overrightarrow{\mathbf{r}}=(\vec{\rho}, z)$.

The integral

$$
I(\vec{\rho}, z) \equiv \int \frac{1-\cos (\overrightarrow{\mathrm{q}} \cdot \overrightarrow{\mathrm{r}})}{q_{\|}^{2}+\lambda^{2} q_{\perp}^{4}} d \overrightarrow{\mathrm{q}}
$$

is evaluated by first integrating over $q_{\|}$using

$$
\int_{-\infty}^{\infty} \frac{1-\cos [a(b-x)]}{x^{2}+c^{2}} d x=\frac{\pi}{c}\left[1-e^{-a c} \cos (a b)\right]
$$

to obtain

$$
\begin{aligned}
& \int_{0}^{\infty} \frac{1-\cos \left(q_{\| z}+\vec{q}_{\perp} \cdot \vec{\rho}\right)}{q_{\|}^{2}+\lambda^{2} q_{\perp}^{4}} d q_{\|} \\
& =\frac{1}{2} \frac{\pi}{\lambda q_{\perp}^{2}}\left[1-\exp \left(-\lambda q_{\perp}^{2} z\right) \cos \left(\overrightarrow{\mathrm{q}}_{\perp} \cdot \overrightarrow{\boldsymbol{\rho}}\right)\right] .
\end{aligned}
$$

Then with $\theta$ being the angle between $\vec{q}_{\perp}$ and $\vec{\rho}$

$$
\begin{aligned}
\int_{0}^{2 \pi}\left[1-\exp \left(-\lambda q_{\perp}^{2} z\right) \cos \left(\overrightarrow{\mathrm{q}}_{\perp} \cdot \vec{\rho}\right)\right] d \theta \\
=2 \pi-\exp \left(-\lambda q_{1}^{2} z\right) \int_{0}^{2 \pi} \cos \left(q_{\perp} \rho \cos \theta\right) d \theta .
\end{aligned}
$$

Using the integral representation of the Bessel function

$$
J_{0}(x)=\frac{1}{\pi} \int_{0}^{\pi} \cos (x \cos \theta) d \theta
$$

we obtain

$I(\vec{\rho}, z)$

$$
=\frac{1}{2} \pi 2 \pi \int_{q_{\min }}^{q_{\max }} \frac{1-\exp \left(-\lambda q_{\perp}^{2} z\right) J_{0}\left(q_{\perp} \rho\right)}{\lambda q_{\perp}^{2}} q_{\perp} d q_{\perp} .
$$

With $q_{\max }=2 \pi / d=q_{0}$ and $q_{\min }=0$ the integral is further evaluated as follows.

One inserts the series expansion form of $J_{0}(x)$

$$
J_{0}(x)=\sum_{n=0} \frac{x^{2 n}(-1)^{n}}{2^{2 n} n ! n !}
$$

and integrates term by term. By comparing the result to the series expansion of the exponential integral

$$
\begin{aligned}
& E_{1}(-x) \equiv \int_{x}^{\infty} e^{-t / t} d t \\
& E_{1}(x)=-\gamma-\ln x-\sum_{n=1}^{\infty} \frac{(-1)^{n} x^{n}}{n(n !)} \\
& (\gamma=\text { Euler's const. }), \text { one finds } \\
& G(\vec{\rho}, z) \sim e^{-2 \eta \gamma}\left(\frac{4 d^{2}}{\rho^{2}}\right) e^{-\eta E_{1}} 1\left(\rho^{2} / 4 \lambda z\right)
\end{aligned}
$$

with $\eta \equiv q_{0}^{2} k T /(8 \pi B \lambda)$. This expression for $G(\vec{\rho}, z)$ has the asymptotic forms given in Eqs. (3a) and $(3 \mathrm{~b})$. 


\section{APPENDIX B: SINGLE FACE VERSUS CHANNEL-CUT CRYSTALS}

In this Appendix we compare for the sake of completeness single face crystals with channel-cut crystals. The conclusions are by and large in agreement with those obtained by Bonse and Hart in their pioneering paper on channel-cut crystals in 1968.

In the left part of Fig. 9 is shown the direct beam profile in the nondispersive orientation (cf. Fig. 3) using the backside of the two channel-cut crystals. We notice that with the open detector geometry used in the experiment the direct beam has long tails falling off as the square of the misset angle, or as $q \|^{-2}$. It would therefore not be possible to conclude from an observed $q_{\|}^{-2}$ falloff from an SmA sample whether it was a resolution effect or a true Landau-Peierls behavior. The $q_{\|}^{-2}$ dependence of the direct beam is a dynamical-diffraction effect due primarily to the finite penetration of the $\mathrm{x}$-ray beam into the perfect crystals. The more of reciprocal space around the Bragg point is picked up by the detector, the larger will the relative weight of the $q_{\|}^{2}$ tails be. Indeed, as also shown in the left part of Fig. 8, when the vertical resolution is improved by about an order of magnitude by horizontal Soller splits in front of the detector, the relative weight of the $q \|^{-2}$ component drops by about an order of magnitude.

However, when the Bragg scattering and the diffuse scattering is tripled in the channel-cut crystals,

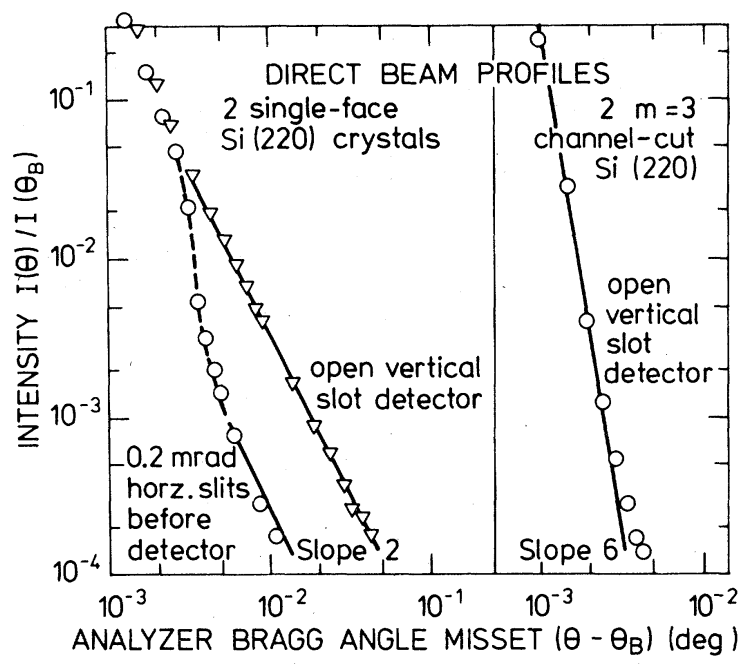

FIG. 9. Direct beam profiles of two $\mathrm{Si}(220)$ crystals in nondispersive orientation. With single reflections (left panel) tails fall off as $\Delta \theta^{-2}$, the tails being more pronounced with relaxed vertical resolution, whereas with triple reflections both in the monochromator and the analyzer channelcut crystals the tails drop off very rapidly as $\Delta \theta^{-6}$.

the Bragg-peak intensity is only diminished to about $50 \%$, but the diffuse $q_{\|}^{-2}$ wings become cubed to $q_{\|}^{-6}$ wings. Indeed, our experiment confirms this simple picture: The log-log plot gives approximately a slope of -6 for the direct beam profile of the channel-cut crystals.
'Permanent address: Dept. of Physics, Massachusetts Institute of Technology, Cambridge, Mass. 02139.

${ }^{1}$ R. E. Peierls, Helv. Phys. Acta Suppl. 7, 81 (1934).

${ }^{2}$ L. D. Landau, in Collected Papers of L. D. Landau, edited by D. ter Haar (Gordon and Breach, New York, 1965), p. 209.

3J. M. Kosterlitz and D. J. Thouless, J. Phys. C $\underline{6}, 118$ (1973)

${ }^{4}$ Very interesting related phenomena are, however, seen in helium films. See, for example, D.J. Bishop and J. D. Reppy, Phys. Rev. Lett. 40, 1727 (1978). Also recent experiments by D. E. Moncton and R. Pindak [Phys. Rev. Lett. 43,701 (1979)] indicate that power-law decay of correlation functions may be observable in smectic- $B$ films.

${ }^{5}$ L. D. Landau and E. M. Lifshitz, Statistical Physics (Addison-Wesley, Reading, Massachusetts, 1969), p. 403.

6P. G. de Gennes, The Physics of Liquid Crystals (Clarendon, Oxford, 1974)

7J. Als-Nielsen, R. J. Birgeneau, M. Kaplan, J. D. Litster, and C. R. Safinya, Phys. Rev. Lett. 39, 1668 (1977).

${ }^{8} \mathrm{H}$. Birecki, R. Schaetzing, F. Rondelez, and J. D. Litster, Phys. Rev. Lett. $\underline{\text { 36, }} 1376$ (1976).
${ }^{9}$ C. A. Schantz and D. L. Johnson, Phys. Rev. A 17,1504 (1978).

${ }^{10}$ B. I. Halperin and T. C. Lubensky, Solid State Commun. 14, 997 (1974).

${ }_{11}$ A. Caillé, C. R. Acad. Sci. Ser. B 274, 891 (1972).

${ }^{12}$ H. -J. Mikeska and H. Schmidt, J. Low Temp. Phys. 2, $371(1970)$

${ }^{13}$ Y. Imry and L. Gunther, Phys. Rev. B $\underline{3}, 3939$ (1971).

${ }^{14}$ Y. Imry, CRC Crit. Rev. Solid State and Mater. Sci. (USA) $\underline{8}, 157$ (1978).

${ }^{15}$ For a highly readable discussion of dynamical diffraction theory see B. E. Warren, X-ray Diffraction (AddisonWesley, Reading, Massachusetts, 1969), Chap. 13.

${ }^{16}$ U. Bonse and M. Hart, Appl. Phys. Lett. 7, 238 (1965).

${ }^{17}$ J. D. Litster, J. Als-Nielsen, R. J. Birgeneau, S. S. Dana, D. Davidov, F. Garcia-Golding, M. Kaplan, C. R. Safinya, and R. Schaetzing, J. Phys. (Paris) 40, C3-339 (1979).

${ }^{18}$ P. P. Karat and N. V. Madhusudana, Mol. Cryst. Liq. Cryst. 47, 21 (1978).

${ }^{19}$ M. A. Laskeen, Philos. Trans. R. Soc. London, Ser. A 256, 357 (1963)

${ }^{20}$ I. H. Ibrahim and W. Haase, J. Phys. (Paris) $\underline{40}$, C6-167 (1979). 